Pacific

Journal of

Mathematics

IRREDUCIBLE REPRESENTATIONS FOR THE ABELIAN EXTENSION OF THE LIE ALGEBRA OF DIFFEOMORPHISMS OF TORI IN DIMENSIONS GREATER THAN 1

\author{
CUIPO JIANG AND QIFEN JIANG
}




\title{
IRREDUCIBLE REPRESENTATIONS FOR THE ABELIAN EXTENSION OF THE LIE ALGEBRA OF DIFFEOMORPHISMS OF TORI IN DIMENSIONS GREATER THAN 1
}

\author{
CUIPO JiAng AND QIFEN JiAnG
}

\begin{abstract}
We classify the irreducible weight modules of the abelian extension of the Lie algebra of diffeomorphisms of tori of dimension greater than 1 , with finite-dimensional weight spaces.
\end{abstract}

\section{Introduction}

Let $W_{v+1}$ be the Lie algebra of diffeomorphisms of the $(v+1)$-dimensional torus. If $v=0$, the universal central extension of the complex Lie algebra $W_{1}$ is the Virasoro algebra, which, together with its representations, plays a very important role in many areas of mathematics and physics [Belavin et al. 1984; Dotsenko and Fateev 1984; Di Francesco et al. 1997]. The representation theory of the Virasoro algebra has been studied extensively; see, for example, [Kac 1982; Kaplansky and Santharoubane 1985; Chari and Pressley 1988; Mathieu 1992].

If $v \geq 1$, however, the Lie algebra $W_{v+1}$ has no nontrivial central extension [Ramos et al. 1990]. But $W_{v+1}$ has abelian extensions whose abelian ideals are the central parts of the corresponding toroidal Lie algebras; see [Berman and Billig 1999], for example. There is a close connection between irreducible integrable modules of the toroidal Lie algebra and irreducible modules of the abelian extension $\mathscr{L}$; see [Berman and Billig 1999; Eswara Rao and Moody 1994; Jiang and Meng 2003], for instance. In fact, the classification of integrable modules of toroidal Lie algebras and their subalgebras depends heavily on the classification of irreducible representations of $\mathscr{L}$ and its subalgebras. See [Billig 2003] for the constructions of the abelian extensions for the group of diffeomorphisms of a torus.

In this paper we study the irreducible weight modules of $\mathscr{L}$, for $v \geq 1$. If $V$ is an irreducible weight module of $\mathscr{L}$ some of whose central charges $c_{0}, \ldots, c_{v}$ are nonzero, one can assume that $c_{0}, \ldots, c_{N}$ are $\mathbb{Z}$-linearly independent and $c_{N+1}=$ $\cdots=c_{v}=0$, where $N \geq 0$. We prove that if $N \geq 1$, then $V$ must have weight

MSC2000: primary 17B67, 17B65; secondary 17B68.

Keywords: irreducible representation, abelian extension, central charge.

Work supported in part by NSF of China, grants No. 10271076 and No. 10571119. 
spaces which are infinite-dimensional. So if all the weight spaces of $V$ are finitedimensional, $N$ vanishes. We classify the irreducible modules of $\mathscr{L}$ with finitedimensional weight spaces and some nonzero central charges. We prove that such a module $V$ is isomorphic to a highest weight module. The highest weight space $T$ is isomorphic to an irreducible $\left(\mathscr{A}_{v}+W_{v}\right)$-module all of whose weight spaces have the same dimension, where $\mathscr{A}_{v}$ is the ring of Laurent polynomials in $v$ commuting variables, regarded as a commutative Lie algebra. An important step is to characterize the $\mathscr{A}_{v}$-module structure of $T$. It turns out that the action of $\mathscr{A}_{v}$ on $T$ is essentially multiplication by polynomials in $\mathscr{A}_{v}$. Therefore $T$ can be identified with Larsson's construction [1992] by a result in [Eswara Rao 2004]. That is, $T$ is a tensor product of $g l_{v}$-module with $\mathscr{A}_{v}$.

When all the central charges of $V$ are zero, we prove that the abelian part acts on $V$ as zero if $V$ is a uniformly bounded $\mathscr{L}$-module. So the result in this case is not complete.

Throughout the paper, $\mathbb{C}, \mathbb{Z}_{+}$and $\mathbb{Z}_{-}$denote the sets of complex numbers, positive integers and negative integers.

\section{Basic concepts and results}

Let $\mathscr{A}_{v+1}=\mathbb{C}\left[t_{0}^{ \pm 1}, t_{1}^{ \pm 1}, \ldots, t_{v}^{ \pm 1}\right](v \geq 1)$ be the ring of Laurent polynomials in commuting variables $t_{0}, t_{1}, \ldots, t_{v}$. For $\underline{n}=\left(n_{1}, n_{2}, \ldots, n_{v}\right) \in \mathbb{Z}^{v}, n_{0} \in \mathbb{Z}$, we denote $t_{0}^{n_{0}} t_{1}^{n_{1}} \cdots t_{v}^{n_{v}}$ by $t_{0}^{n_{0}} t^{n}$. Let $\tilde{\mathscr{T}}$ be the free $\mathscr{A}_{v+1}$-module with basis $\left\{k_{0}, k_{1}, \ldots, k_{v}\right\}$ and let $d \tilde{\mathscr{K}}$ be the subspace spanned by all elements of the form

$$
\sum_{i=0}^{v} r_{i} t_{0}^{r_{0}} t^{\underline{r}} k_{i}, \quad \text { for }\left(r_{0}, \underline{r}\right)=\left(r_{0}, r_{1}, \ldots, r_{v}\right) \in \mathbb{Z}^{v+1} .
$$

Set $\mathscr{K}=\tilde{\mathscr{K}} / d \tilde{\mathscr{K}}$ and denote the image of $t_{0}^{r_{0}} t^{r} k_{i}$ still by itself. Then $\mathscr{K}$ is spanned by the elements $\left\{t_{0}^{r_{0}} t^{\underline{r}} k_{p} \mid p=0,1, \ldots, v, r_{0} \in \mathbb{Z}, \underline{r} \in \mathbb{Z}^{v}\right\}$ with relations

$$
\sum_{p=0}^{v} r_{p} t_{0}^{r_{0}} t^{\underline{r}} k_{p}=0
$$

Let $\mathscr{D}$ be the Lie algebra of derivations on $\mathscr{A}_{v+1}$. Then

$$
\mathscr{D}=\left\{\sum_{p=0}^{v} f_{p}\left(t_{0}, t_{1}, \ldots, t_{\nu}\right) d_{p} \mid f_{p}\left(t_{0}, t_{1}, \ldots, t_{\nu}\right) \in \mathscr{A}_{\nu+1}\right\},
$$

where $d_{p}=t_{p} \partial / \partial t_{p}, p=0,1, \ldots, v$. From [Berman and Billig 1999] we know that the algebra $\mathscr{D}$ admits two nontrivial 2-cocycles with values in $\mathscr{K}$ :

$$
\tau_{1}\left(t_{0}^{m_{0}} t^{\underline{m}} d_{a}, t_{0}^{n_{0}} t^{\underline{n}} d_{b}\right)=-n_{a} m_{b} \sum_{p=0}^{\nu} m_{p} t_{0}^{m_{0}+n_{0}} t^{\underline{m}+\underline{n}} k_{p},
$$




$$
\tau_{2}\left(t_{0}^{m_{0}} t^{\underline{m}} d_{a}, t_{0}^{n_{0}} t^{\underline{n}} d_{b}\right)=m_{a} n_{b} \sum_{p=0}^{v} m_{p} t_{0}^{m_{0}+n_{0}} t^{\underline{m}+\underline{n}} k_{p} .
$$

Let $\tau=\mu_{1} \tau_{1}+\mu_{2} \tau_{2}$ be an arbitrary linear combination of $\tau_{1}$ and $\tau_{2}$. Then the corresponding abelian extension of $\mathscr{D}$ is

$$
\mathscr{L}=\mathscr{D} \oplus \mathscr{K},
$$

with the Lie bracket

$$
\begin{aligned}
& {\left[t_{0}^{m_{0}} t^{\underline{m}} d_{a}, t_{0}^{n_{0}} t^{\underline{n}} k_{b}\right]=n_{a} t_{0}^{m_{0}+n_{0}} t^{\underline{m}+\underline{n}} k_{b}+\delta_{a b} \sum_{p=0}^{v} m_{p} t_{0}^{m_{0}+n_{0}} t^{\underline{m}+\underline{n}} k_{p},} \\
& {\left[t_{0}^{m_{0}} t^{\underline{m}} d_{a}, t_{0}^{n_{0}} t^{\underline{n}} d_{b}\right]=n_{a} t_{0}^{m_{0}+n_{0}} t^{\underline{m}+\underline{n}} d_{b}-m_{b} t_{0}^{m_{0}+n_{0}} t^{\underline{m}+\underline{n}} d_{a}} \\
& +\tau\left(t_{0}^{m_{0}} t^{\underline{m}} d_{a}, t_{0}^{n_{0}} t^{\underline{n}} d_{b}\right) .
\end{aligned}
$$

The sum

$$
\mathfrak{h}=\left(\bigoplus_{i=0}^{v} \mathbb{C} k_{i}\right) \oplus\left(\bigoplus_{i=0}^{v} \mathbb{C} d_{i}\right)
$$

is an abelian Lie subalgebra of $\mathscr{L}$. An $\mathscr{L}$-module $V$ is called a weight module if

$$
V=\bigoplus_{\lambda \in \mathfrak{h}^{*}} V_{\lambda}
$$

where $V_{\lambda}=\{v \in V \mid h \cdot v=\lambda(h) v$ for all $h \in \mathfrak{h}\}$. Denote by $P(V)$ the set of all weights. Throughout the paper, we assume that $V$ is an irreducible weight module of $\mathscr{L}$ with finite-dimensional weight spaces. Since $V$ is irreducible, we have

$$
\left.k_{i}\right|_{V}=c_{i}
$$

where the constants $c_{i}$, for $i=0,1, \ldots, v$, are called the central charges of $V$.

Lemma 2.1. Let $A=\left(a_{i j}\right)(0 \leq i, j \leq v)$ be $a(v+1) \times(v+1)$ matrix such that $\operatorname{det} A=1$ and $a_{i j} \in \mathbb{Z}$. There exists an automorphism $\sigma$ of $\mathscr{L}$ such that

$$
\sigma\left(t^{\bar{m}} k_{j}\right)=\sum_{p=0}^{v} a_{p j} t^{\bar{m} A^{T}} k_{p}, \quad \sigma\left(t^{\bar{m}} d_{j}\right)=\sum_{p=0}^{v} b_{j p} t^{\bar{m} A^{T}} d_{p}, \quad 0 \leq j \leq v
$$

where $t^{\bar{m}}=t_{0}^{m_{0}} t^{\underline{m}}, B=\left(b_{i j}\right)=A^{-1}$.

\section{The structure of $V$ with nonzero central charges}

In this section, we discuss the weight module $V$ which has nonzero central charges. It follows from Lemma 2.1 that we can assume that $c_{0}, c_{1}, \ldots, c_{N}$ are $\mathbb{Z}$-linearly independent, i.e., if $\sum_{i=0}^{N} a_{i} c_{i}=0, a_{i} \in \mathbb{Z}$, then all $a_{i}(i=0, \ldots, N)$ must be zero, 
and $c_{N+1}=c_{N+2}=\cdots=c_{v}=0$, where $N \geq 0$. For $\bar{m}=\left(m_{0}, \underline{m}\right)$, denote $t_{0}^{m_{0}} t^{\underline{m}}$ by $t^{\bar{m}}$ as in Lemma 2.1. It is easy to see that $V$ has the decomposition

$$
V=\bigoplus_{\bar{m} \in \mathbb{Z}^{v+1}} V_{\bar{m}}
$$

where $V_{\bar{m}}=\left\{v \in V \mid d_{i}(v)=\left(\gamma_{0}\left(d_{i}\right)+m_{i}\right) v, i=0,1, \ldots, v\right\}$, with $\gamma_{0} \in P(V)$ a fixed weight, and $\bar{m}=\left(m_{0}, m_{1}, \ldots, m_{v}\right) \in \mathbb{Z}^{v+1}$. If $V$ has finite-dimensional weight spaces, the $V_{\bar{m}}$ are finite-dimensional, for $\bar{m} \in \mathbb{Z}^{v+1}$.

\section{In Lemmas 3.1-3.6 we assume that V has finite-dimensional weight spaces.}

Lemma 3.1. For $p \in\{0,1, \ldots, v\}$ and $0 \neq t^{\bar{m}} k_{p} \in \mathscr{L}$, if there is a nonzero element $v$ in $V$ such that $t^{\bar{m}} k_{p} v=0$, then $t^{\bar{m}} k_{p}$ is locally nilpotent on $V$.

Lemma 3.2. Let $t_{0}^{m_{0}} t^{\underline{m}} k_{p} \in \mathscr{L}$ be such that $\bar{m}=\left(m_{0}, \underline{m}\right) \neq \overline{0}$, and there exists $0 \leq a \leq N$ such that $m_{a} \neq 0$ if $N<p \leq v$. If $t_{0}^{m_{0}} t^{\underline{m}} k_{p}$ is locally nilpotent on $V$, then $\operatorname{dim} V_{\bar{n}}>\operatorname{dim} V_{\bar{n}+\bar{m}}$ for all $\bar{n} \in \mathbb{Z}^{\nu+1}$.

Proof. Case 1: $p \in\{0,1, \ldots, N\}$. We first prove that $\operatorname{dim} V_{\bar{n}} \geq \operatorname{dim} V_{\bar{n}+\bar{m}}$ for all $\bar{n} \in \mathbb{Z}^{v+1}$. Suppose $\operatorname{dim} V_{\bar{n}}=m, \operatorname{dim} V_{\bar{n}+\bar{m}}=n$. Let $\left\{w_{1}, w_{2}, \ldots, w_{n}\right\}$ be a basis of $V_{\bar{n}+\bar{m}}$ and $\left\{w_{1}^{\prime}, w_{2}^{\prime}, \ldots, w_{m}^{\prime}\right\}$ a basis of $V_{\bar{n}}$. We can assume that $m_{a} \neq 0$ for some $0 \leq a \leq v$ distinct from $p$, where $\bar{m}=\left(m_{0}, \underline{m}\right)=\left(m_{0}, m_{1}, \ldots, m_{v}\right)$. Since $t^{\bar{m}} k_{p}$ is locally nilpotent on $V$ and $V_{\bar{n}+\bar{m}}$ is finite-dimensional, there exists $k>0$ such that $\left(t^{\bar{m}} k_{p}\right)^{k} V_{\bar{n}+\bar{m}}=0$. Therefore

$$
\left(t^{-\bar{m}} d_{a}\right)^{k}\left(t^{\bar{m}} k_{p}\right)^{k}\left(w_{1}, w_{2}, \ldots, w_{n}\right)=0 .
$$

On the other hand, by induction on $k$, we can deduce that

$$
\left(t^{-\bar{m}} d_{a}\right)^{k}\left(t^{\bar{m}} k_{p}\right)^{k}=\sum_{i=0}^{k} \frac{k ! k !}{i !(k-i) !(k-i) !} m_{a}^{i} c_{p}^{i}\left(t^{\bar{m}} k_{p}\right)^{k-i}\left(t^{-\bar{m}} d_{a}\right)^{k-i} .
$$

Therefore

$$
\begin{array}{r}
t^{\bar{m}} k_{p}\left(\sum_{i=0}^{k-1} \frac{k ! k !}{i !(k-i) !(k-i) !} m_{a}^{i} c_{p}^{i}\left(t^{\bar{m}} k_{p}\right)^{k-1-i}\left(t^{-\bar{m}} d_{a}\right)^{k-1-i}\right) t^{-\bar{m}} d_{a}\left(w_{1}, w_{2}, \ldots, w_{n}\right) \\
=-k ! m_{a}^{k} c_{p}^{k}\left(w_{1}, w_{2}, \ldots, w_{n}\right) .
\end{array}
$$

Assume that

$$
\begin{array}{r}
\left(\sum_{i=0}^{k-1} \frac{k ! k !}{i !(k-i) !(k-i) !} m_{a}^{i} c_{p}^{i}\left(t^{\bar{m}} k_{p}\right)^{k-1-i}\left(t^{-\bar{m}} d_{a}\right)^{k-1-i}\right) t^{-\bar{m}} d_{a}\left(w_{1}, w_{2}, \ldots, w_{n}\right) \\
=\left(w_{1}^{\prime}, w_{2}^{\prime}, \ldots, w_{m}^{\prime}\right) C,
\end{array}
$$

with $C \in \mathbb{C}^{m \times n}$, and that

$$
t^{\bar{m}} k_{p}\left(w_{1}^{\prime}, w_{2}^{\prime}, \ldots, w_{m}^{\prime}\right)=\left(w_{1}, w_{2}, \ldots, w_{n}\right) B
$$


with $B \in \mathbb{C}^{n \times m}$. Then

$$
B C=-k ! m_{a}^{k} c_{p}^{k} I .
$$

This implies that $m \geq n$. So $\operatorname{dim} V_{\bar{n}} \geq \operatorname{dim} V_{\bar{n}+\bar{m}}$ for all $\bar{n} \in \mathbb{Z}^{v+1}$. Also, by (3-1) and the fact that $r(B)=n$, we know that $m>n$ if and only if there exists $v \in V_{\bar{n}}$ such that $t^{\bar{m}} k_{p} \cdot v=0$. Since $t^{\bar{m}} k_{p}$ is locally nilpotent on $V$, there exist an integer $s \geq 0$ and $w \in V_{\bar{n}+s \bar{m}}$ such that

$$
\left(t^{\bar{m}} k_{p}\right) \cdot w=0 .
$$

Therefore $\left(t^{-\bar{m}} k_{p}\right) t^{\bar{m}} k_{p} \cdot w=t^{\bar{m}} k_{p}\left(t^{-\bar{m}} k_{p} \cdot w\right)=0$. If $t^{-\bar{m}} k_{p} \cdot w=0$, by the proof above, $\operatorname{dim} V_{\bar{n}+s \bar{m}-\bar{m}}<\operatorname{dim} V_{\bar{n}+s \bar{m}}$, contradicting the fact that $\operatorname{dim} V_{\bar{n}+s \bar{m}-\bar{m}} \geq$ $\operatorname{dim} V_{\bar{n}+s \bar{m}}$. Therefore $\left(t^{-\bar{m}} k_{p}\right)^{r} \cdot w \neq 0$ for all $r \in \mathbb{N}$. Since

$$
\left(t^{-\bar{m}} k_{p}\right)^{s} t^{\bar{m}} k_{p} \cdot w=t^{\bar{m}} k_{p}\left(t^{-\bar{m}} k_{p}\right)^{s} \cdot w=0
$$

and $\left(t^{-\bar{m}} k_{p}\right)^{s} \cdot w \in V_{\bar{n}}$, it follows that there is a nonzero element $v$ in $V_{\bar{n}}$ such that $t^{\bar{m}} k_{p} \cdot v=0$. Thus $n<m$.

Case 2: $N<p \leq v$. The proof is similar to that of case 1, but we have to consider $\overline{t^{-\bar{m}} d_{p}}$ and $t^{\bar{m}} k_{p}$ instead and use the $\mathbb{Z}$-linear independence of $c_{1}, \ldots, c_{N}$.

Lemma 3.3. Let $0 \neq t^{\bar{m}} k_{p} \in \mathscr{L}$ and $0 \neq t^{\bar{n}} k_{p} \in \mathscr{L}$ be such that $\left(m_{0}, \ldots, m_{N}\right) \neq 0$, $\left(n_{0}, \ldots, n_{N}\right) \neq 0$ if $N<p \leq v$, where $\bar{m}=\left(m_{0}, m_{1}, \ldots, m_{v}\right)$.

(1) If $t^{\bar{m}} k_{p}$ is locally nilpotent on $V, t^{\bar{m}} k_{q}$ is locally nilpotent for $q=0,1, \ldots, v$.

(2) If both $0 \neq t^{\bar{m}} k_{p}$ and $0 \neq t^{\bar{n}} k_{p}$ are locally nilpotent on $V$, then $t^{\bar{m}+\bar{n}} k_{p}$ is locally nilpotent.

(3) If $0 \neq t^{\bar{m}+\bar{n}} k_{p}$ is locally nilpotent on $V$ and $\left(m_{0}+n_{0}, \ldots, m_{N}+n_{N}\right) \neq 0$ if $N<p \leq v$, then $t^{\bar{m}} k_{p}$ or $t^{\bar{n}} k_{p}$ is locally nilpotent.

Lemma 3.4. For $0 \leq p \leq v$, let $0 \neq t^{\bar{m}} k_{p} \in \mathscr{L}$ be such that $\left(m_{0}, \ldots, m_{N}\right) \neq 0$, where $\bar{m}=\left(m_{0}, m_{1}, \ldots, m_{v}\right)$. Then $t^{\bar{m}} k_{p}$ or $t^{-\bar{m}} k_{p}$ is locally nilpotent on $V$.

Proof. The proof occupies the next few pages. We first deal with the case $0 \leq p \leq N$. Without losing generality, we can take $p=0$.

Suppose the lemma is false. By Lemma 3.2, for any $\bar{r} \in \mathbb{Z}^{v+1}$ we have

$$
\operatorname{dim} V_{\bar{r}+\bar{m}}=\operatorname{dim} V_{\bar{r}}=\operatorname{dim} V_{\bar{r}-\bar{m}}, \quad t^{\bar{m}} k_{0} V_{\bar{r}}=V_{\bar{r}+\bar{m}}, \quad t^{-\bar{m}} k_{0} V_{\bar{r}}=V_{\bar{r}-\bar{m}} .
$$

Fix $\bar{r}=\left(r_{0}, \underline{r}\right) \in \mathbb{Z}^{\nu+1}$ such that $V_{\bar{r}} \neq 0$. Let $\left\{v_{1}, \ldots, v_{n}\right\}$ be a basis of $V_{\bar{r}}$ and set

$$
v_{i}(k \bar{m})=\frac{1}{c_{0}} t^{k \bar{m}} k_{0} \cdot v_{i}, \quad i=1,2, \ldots, n,
$$


where $k \in \mathbb{Z} \backslash\{0\}$. Then $\left\{v_{1}(k \bar{m}), v_{2}(k \bar{m}), \ldots, v_{n}(k \bar{m})\right\}$ is a basis of $V_{\bar{r}+k \bar{m}}$. Let $B_{-\bar{m}, \bar{m}}^{(0)}, B_{\bar{m},-\bar{m}}^{(0)} \in \mathbb{C}^{n \times n}$ be such that

$$
\begin{aligned}
\frac{1}{c_{0}} t^{\bar{m}} k_{0}\left(v_{1}(-\bar{m}), v_{2}(-\bar{m}), \ldots, v_{n}(-\bar{m})\right) & =\left(v_{1}, v_{2}, \ldots, v_{n}\right) B_{\bar{m},-\bar{m}}^{(0)}, \\
\frac{1}{c_{0}} t^{-\bar{m}} k_{0}\left(v_{1}(\bar{m}), v_{2}(\bar{m}), \ldots, v_{n}(\bar{m})\right) & =\left(v_{1}, v_{2}, \ldots, v_{n}\right) B_{-\bar{m}, \bar{m}}^{(0)} .
\end{aligned}
$$

Since $t^{\bar{m}} k_{0}$ and $t^{-\bar{m}} k_{0}$ are commutative, it is easy to deduce that

$$
B_{\bar{m},-\bar{m}}^{(0)}=B_{-\bar{m}, \bar{m}}^{(0)} \cdot
$$

By Lemma 3.1, $B_{\bar{m},-\bar{m}}^{(0)}$ is an $n \times n$ invertible matrix.

Claim. $B_{\bar{m},-\bar{m}}^{(0)}$ does not have distinct eigenvalues.

Proof. Set $c=1 / c_{0}$. To prove the claim, we need to consider $c t^{\bar{m}} k_{0} c t^{-\bar{m}} k_{0}-\lambda$ id, where $\lambda \in \mathbb{C}^{*}$. As in the proof of Lemma 3.1, we can deduce that if there is a nonzero element $v$ in $V$ such that $\left(c t^{\bar{m}} k_{0} c t^{-\bar{m}} k_{0}-\lambda\right.$ id $) v=0$, then $c t^{\bar{m}} k_{0} c t^{-\bar{m}} k_{0}-$ $\lambda$ id is locally nilpotent on $V$. On the other hand, we have

$$
\left(c t^{\bar{m}} k_{0} c t^{-\bar{m}} k_{0}-\lambda \mathrm{id}\right)^{l}\left(v_{1}, v_{2}, \ldots, v_{n}\right)=\left(v_{1}, v_{2}, \ldots, v_{n}\right)\left(B_{\bar{m},-\bar{m}}^{(0)}-\lambda \mathrm{id}\right)^{l} .
$$

Therefore the claim holds.

For $p \in\{1,2, \ldots, v\}$, let $C_{\bar{m}, \overline{0}}^{p}, C_{\bar{m},-\bar{m}}^{p} \in \mathbb{C}^{n \times n}$ be such that

$$
\begin{aligned}
& t^{\bar{m}} k_{p}\left(v_{1}, v_{2}, \ldots, v_{n}\right)=\left(v_{1}(\bar{m}), \ldots, v_{n}(\bar{m})\right) C_{\bar{m}, \overline{0}}^{(p)}, \\
& t^{\bar{m}} k_{p}\left(v_{1}(-\bar{m}), \ldots, v_{n}(-\bar{m})\right)=\left(v_{1}, v_{2}, \ldots, v_{n}\right) C_{\bar{m},-\bar{m}}^{(p)} .
\end{aligned}
$$

Since

$$
\frac{1}{c_{0}} t^{-\bar{m}} k_{0} t^{\bar{m}} k_{p}\left(v_{1}, v_{2}, \ldots, v_{n}\right)=t^{\bar{m}} k_{p} \frac{1}{c_{0}} t^{-\bar{m}} k_{0}\left(v_{1}, v_{2}, \ldots, v_{n}\right),
$$

we have

$$
C_{\bar{m},-\bar{m}}^{(p)}=B_{-\bar{m}, \bar{m}}^{(0)} C_{\bar{m}, \overline{0}}^{(p)} .
$$

Furthermore, by the fact that

$$
\frac{1}{c_{0}} t^{\bar{m}} k_{0} \frac{1}{c_{0}} t^{-\bar{m}} k_{0} t^{\bar{m}} k_{p}\left(v_{1}, v_{2}, \ldots, v_{n}\right)=t^{\bar{m}} k_{p} \frac{1}{c_{0}} t^{\bar{m}} k_{0} \frac{1}{c_{0}} t^{-\bar{m}} k_{0}\left(v_{1}, v_{2}, \ldots, v_{n}\right)
$$

and

$$
t^{\bar{m}} k_{q} \frac{1}{c_{0}} t^{-\bar{m}} k_{0} t^{\bar{m}} k_{p}=t^{\bar{m}} k_{p} \frac{1}{c_{0}} t^{-\bar{m}} k_{0} t^{\bar{m}} k_{q}
$$


we deduce that

$$
B_{-\bar{m}, \bar{m}}^{(0)} C_{\bar{m}, \overline{0}}^{(p)}=C_{\bar{m}, \overline{0}}^{(p)} B_{-\bar{m}, \bar{m}}^{(0)}, \quad C_{\bar{m}, \overline{0}}^{(p)} C_{\bar{m}, \overline{0}}^{(q)}=C_{\bar{m}, \overline{0}}^{(q)} C_{\bar{m}, \overline{0}}^{(p)}, \quad 1 \leq p, q \leq v .
$$

Hence there exists $D \in \mathbb{C}^{n \times n}$ such that $\left\{D^{-1} B_{-\bar{m}, \bar{m}}^{(0)} D, D^{-1} C_{\bar{m}, \overline{0}}^{(p)} D \mid 1 \leq p \leq \nu\right\}$ are all upper triangular matrices. If we set

$$
\left(w_{1}, w_{2}, \ldots, w_{n}\right)=\left(v_{1}, v_{2}, \ldots, v_{n}\right) D
$$

and

then

$$
w_{i}(k \bar{m})=\frac{1}{c_{0}} t^{k \bar{m}} k_{0} w_{i}, 1 \leq i \leq n, k \in \mathbb{Z} \backslash\{0\},
$$

$$
\begin{gathered}
\frac{1}{c_{0}} t^{k \bar{m}} k_{0}\left(w_{1}(-\bar{m}), w_{2}(-\bar{m}), \ldots, w_{n}(-\bar{m})\right)=\left(w_{1}, \ldots, w_{n}\right) D^{-1} B_{-\bar{m}, \bar{m}}^{(0)} D, \\
t^{\bar{m}} k_{p}\left(w_{1}, w_{2}, \ldots, w_{n}\right)=\left(w_{1}(\bar{m}), \ldots, w_{n}(\bar{m})\right) D^{-1} C_{\bar{m}, \overline{0}}^{(p)} D .
\end{gathered}
$$

So we can assume that $B_{-\bar{m}, \bar{m}}^{(0)}, C_{\bar{m}, \overline{0}}^{(p)}$, and $C_{\bar{m},-\bar{m}}^{(p)}$, for $1 \leq p \leq v$ are all invertible upper triangular matrices. Furthermore, because

$$
\left(t^{\bar{m}} k_{p} \frac{1}{c_{0}} t^{-\bar{m}} k_{0}-\lambda \mathrm{id}\right)^{l}\left(v_{1}, v_{2}, \ldots, v_{n}\right)=\left(v_{1}, v_{2}, \ldots, v_{n}\right)\left(C_{\bar{m},-\bar{m}}^{(p)}-\lambda \mathrm{id}\right)^{l},
$$

the argument used in the proof of the claim shows that $C_{\bar{m},-\bar{m}}^{(p)}$ also does not have distinct eigenvalues. For $1 \leq p \leq N$, set

$$
B_{\bar{m},-\bar{m}}^{(p)}=\frac{1}{c_{p}} C_{\bar{m},-\bar{m}}^{(p)}
$$

and for $0 \leq p \leq N$ denote by $\lambda_{p}$ the eigenvalue of $B_{\bar{m},-\bar{m}}^{(p)}$.

Let $A_{k \bar{m}, \overline{0}}^{(a)}$ and $A_{k_{1} \bar{m}, k_{2} \bar{m}}^{(a)}$, for $0 \leq a \leq v$ and $k, k_{1}, k_{2} \in \mathbb{Z} \backslash\{0\}$, be such that

$$
\begin{aligned}
& t^{k \bar{m}} d_{a}\left(v_{1}, v_{2}, \ldots, v_{n}\right)=\left(v_{1}(k \bar{m}), v_{2}(k \bar{m}), \ldots, v_{n}(k \bar{m})\right) A_{k \bar{m}, \overline{0}}^{(a)}, \\
& t^{k_{1} \bar{m}} d_{a}\left(v_{1}\left(k_{2} \bar{m}\right), v_{2}\left(k_{2} \bar{m}\right), \ldots, v_{n}\left(k_{2} \bar{m}\right)\right) \\
& =\left(v_{1}\left(k_{1} \bar{m}+k_{2} \bar{m}\right), \ldots, v_{n}\left(k_{1} \bar{m}+k_{2} \bar{m}\right)\right) A_{k_{1} \bar{m}, k_{2} \bar{m}}^{(a)} .
\end{aligned}
$$

Case 1: $v>1$. Since $t^{\bar{m}} k_{0}=t_{0}^{m_{0}} t^{\bar{m}} k_{0} \neq 0$, it follows that there exists $1 \leq a \leq v$ such that $m_{a} \neq 0$, where $\underline{m}=\left(m_{1}, m_{2}, \ldots, m_{v}\right)$. Let $b \in\{1, \ldots, v\}$ be such that $a \neq b$. Consider

$$
\left[t^{-\bar{m}} d_{a}, \frac{1}{c_{0}} t^{\bar{m}} k_{0}\right]=m_{a} \frac{1}{c_{0}} k_{0}, \quad\left[t^{-\bar{m}} d_{a}, t^{\bar{m}} k_{b}\right]=m_{a} k_{b} .
$$

Case 1.1: There exists $b \in\{0,1, \ldots, v\}$ such that $b \neq 0, a$ and $c_{b}=0$. Then

$$
A_{-\bar{m}, \bar{m}}^{(a)}=B_{\bar{m},-\bar{m}}^{(0)} A_{-\bar{m}, \overline{0}}^{(a)}+m_{a} I, \quad A_{-\bar{m}, \bar{m}}^{(a)} C_{\bar{m}, \overline{0}}^{(b)}=C_{\bar{m},-\bar{m}}^{(b)} A_{-\bar{m}, \overline{0}}^{(a)} .
$$


By (3-2) and (3-3),

$$
A_{-\bar{m}, \overline{0}}^{(a)}+m_{a} B_{\bar{m},-\bar{m}}^{(0)}{ }^{-1}=C_{\bar{m}, \overline{0}}^{(b)} A_{-\bar{m}, \overline{0}}^{(a)} C_{\bar{m}, \overline{0}}^{(b)}{ }^{-1} .
$$

But the sum on the left-hand side cannot be similar to $A_{-\bar{m}, \overline{0}}^{(a)}$, since $m_{a} \neq 0$ and $B_{\bar{m},-\bar{m}}^{(0)}-1$ is an invertible upper triangular matrix and does not have different eigenvalues. Thus this case is excluded.

Case 1.2: $c_{b} \neq 0$ for all $b \in\{0,1, \ldots, v\}, b \neq 0, a$. By (3-4) and (3-2), we have

$$
\begin{aligned}
B_{\bar{m},-\bar{m}}^{(0)} A_{-\bar{m}, \overline{0}}^{(a)} B_{\bar{m},-\bar{m}}^{(0)}+m_{a} B_{\bar{m},-\bar{m}}^{(0)}-m_{a} B_{\bar{m},-\bar{m}}^{(b)}{ }^{-1} & =B_{\bar{m},-\bar{m}}^{(0)} C_{\bar{m}, \overline{0}}^{(b)} A_{-\bar{m}, \overline{0}}^{(a)} C_{\bar{m}, \overline{0}}^{(b)}{ }^{-1} B_{\bar{m},-\bar{m}}^{(0)}{ }^{-1} .
\end{aligned}
$$

(I) There exists $b \neq 0$ and $a$ such that $\lambda_{0} \neq \lambda_{b}$. Then $m_{a} B_{\bar{m},-\bar{m}}^{(0)}-m_{a} B_{\bar{m},-\bar{m}}^{(b)}{ }^{-1}$ is an invertible upper triangular matrix and does not have different eigenvalues. As in case 1.1 , we deduce a contradiction.

(II) $\lambda_{0}=\lambda_{b}$ for all $b \in\{1, \ldots, v\}$ distinct from $a$.

(II.1) Suppose first that $c_{a}=0$ (in this case $N=v-1, a=v$ ) or $c_{a} \neq 0$ and $\lambda_{a}=\lambda_{0}$ (in this case $N=v$ ). Since $\sum_{p=0}^{v} m_{p} t^{\bar{m}} k_{p}=0$, we have

$$
\sum_{p=0}^{v} m_{p} t^{\bar{m}} k_{p} \frac{1}{c_{0}} t^{-\bar{m}} k_{0}=0 .
$$

So $\sum_{p=0}^{\nu} m_{p} C_{\bar{m},-\bar{m}}^{(p)}=0$, and therefore

$$
\sum_{p=0}^{v} m_{p} c_{p}=0,
$$

which contradicts the assumption that $c_{0}, \ldots, c_{N}$ are $\mathbb{Z}$-linearly independent.

(II.2) Now suppose $c_{a} \neq 0, \lambda_{a} \neq \lambda_{0}$ and there exists $b \neq 0$ and $a$ such that $m_{b} \neq 0$. We deduce a contradiction as in case 1.2(I) by interchanging $a$ by $b$.

(II.3) Suppose $c_{a} \neq 0, \lambda_{a} \neq \lambda_{0}$ and $m_{b}=0$ for all $b \in\{1, \ldots, v\}$ distinct from $a$. Then $m_{0} c_{0} \lambda_{0}+m_{a} c_{a} \lambda_{a}=0$. The proof of this case is the same as in case 2.2 below.

Case 2.: $v=1$. In this case $a=1$.

Case 2.1: $c_{a}=0$. Since $\left[t^{-\bar{m}} d_{0}, t^{\bar{m}} k_{0}\right]=\left[t^{-\bar{m}} k_{0}, t^{\bar{m}} d_{0}\right]=0$, we have

$$
A_{-\bar{m}, \bar{m}}^{(0)}=B_{\bar{m},-\bar{m}}^{(0)} A_{-\bar{m}, \overline{0}}^{(0)}, \quad A_{\bar{m},-\bar{m}}^{(0)}=B_{-\bar{m}, \bar{m}}^{(0)} A_{\bar{m}, \overline{0}}^{(0)} .
$$

Therefore

$$
\left[t^{-\bar{m}} d_{0}, t^{\bar{m}} d_{0}\right]\left(v_{1}, v_{2}, \ldots, v_{n}\right)=\left(v_{1}, v_{2}, \ldots, v_{n}\right) B_{-\bar{m}, \bar{m}}^{(0)}\left[A_{-\bar{m}, \overline{0}}^{(0)}, A_{\bar{m}, \overline{0}}^{(0)}\right]
$$


At the same time, we have

$$
\left[t^{-\bar{m}} d_{0}, t^{\bar{m}} d_{0}\right]=2 m_{0} d_{0}+m_{0}^{2}\left(-\mu_{1}+\mu_{2}\right)\left(m_{0} k_{0}+m_{1} k_{1}\right),
$$

where $\tau=\mu_{1} \tau_{1}+\mu_{2} \tau_{2}$ as above. So

$$
B_{-\bar{m}, \bar{m}}^{(0)}\left[A_{-\bar{m}, \overline{0}}^{(0)}, A_{\bar{m}, \overline{0}}^{(0)}\right]=\left(2 m_{0}\left(\gamma_{0}\left(d_{0}\right)+r_{0}\right)+m_{0}^{2}\left(-\mu_{1}+\mu_{2}\right)\left(m_{0} c_{0}+m_{1} c_{1}\right)\right) I,
$$

where $\gamma_{0}$ is the weight fixed above. Since $\gamma_{0}$ is arbitrary, we can choose it such that

$$
2 m_{0}\left(\gamma_{0}\left(d_{0}\right)+r_{0}\right)+m_{0}^{2}\left(-\mu_{1}+\mu_{2}\right)\left(m_{0} c_{0}+m_{1} c_{1}\right) \neq 0 .
$$

But $B_{-\bar{m}, \bar{m}}^{(0)}$ is an invertible triangular matrix and does not have different eigenvalues, in contradiction with (3-5).

Case 2.2: $c_{a} \neq 0$. Since

$$
\begin{aligned}
& {\left[t^{-\bar{m}} d_{0}, t^{\bar{m}} k_{0}\right]=-m_{1} k_{1},\left[t^{-\bar{m}} d_{1}, t^{\bar{m}} k_{0}\right]=m_{1} k_{0} \text { and }} \\
& {\left[t^{\bar{m}} d_{0}, t^{-\bar{m}} k_{0}\right]=m_{1} k_{1},\left[t^{\bar{m}} d_{1}, t^{-\bar{m}} k_{0}\right]=-m_{1} k_{0},}
\end{aligned}
$$

we have

$$
\left[k_{0} t^{-\bar{m}} d_{0}+k_{1} t^{-\bar{m}} d_{1}, t^{\bar{m}} k_{0}\right]=\left[k_{0} t^{\bar{m}} d_{0}+k_{1} t^{\bar{m}} d_{1}, t^{-\bar{m}} k_{0}\right]=0 .
$$

Therefore

$$
\begin{aligned}
& k_{0} A_{-\bar{m}, \bar{m}}^{(0)}+k_{1} A_{-\bar{m}, \bar{m}}^{(1)}=B_{\bar{m},-\bar{m}}^{(0)}\left(k_{0} A_{-\bar{m}, \overline{0}}^{(0)}+k_{1} A_{-\bar{m}, \overline{0}}^{(1)}\right), \\
& k_{0} A_{\bar{m},-\bar{m}}^{(0)}+k_{1} A_{\bar{m},-\bar{m}}^{(1)}=B_{-\bar{m}, \bar{m}}^{(0)}\left(k_{0} A_{\bar{m}, \overline{0}}^{(0)}+k_{1} A_{\bar{m}, \overline{0}}^{(1)}\right),
\end{aligned}
$$

and

$$
\begin{aligned}
{\left[k_{0} t^{-\bar{m}} d_{0}+k_{1} t^{-\bar{m}} d_{1},\right.} & \left.k_{0} t^{\bar{m}} d_{0}+k_{1} t^{\bar{m}} d_{1}\right]\left(v_{1}, \ldots, v_{n}\right) \\
& =\left(v_{1}, \ldots, v_{n}\right) B_{\bar{m},-\bar{m}}^{(0)}\left[k_{0} A_{-\bar{m}, \overline{0}}^{(0)}+k_{1} A_{-\bar{m}, \overline{0}}^{(1)}, k_{0} A_{\bar{m}, \overline{0}}^{(0)}+k_{1} A_{\bar{m}, \overline{0}}^{(1)}\right] .
\end{aligned}
$$

At the same time, we have

$$
\begin{aligned}
{\left[k_{0} t^{-\bar{m}} d_{0}+k_{1} t^{-\bar{m}} d_{1}, k_{0} t^{\bar{m}} d_{0}+k_{1} t^{\bar{m}} d_{1}\right] } \\
\quad=2\left(m_{0} c_{0}+m_{1} c_{1}\right)\left(c_{0} d_{0}+c_{1} d_{1}\right)-\left(m_{0} c_{0}+m_{1} c_{1}\right)^{3}\left(\mu_{1}-\mu_{2}\right) \text { id. }
\end{aligned}
$$

Since $c_{0}$ and $c_{1}$ are $\mathbb{Z}$-linearly independent, we know that $m_{0} c_{0}+m_{1} c_{1} \neq 0$. As in case 2.1 , we deduce a contradiction.

This concludes the first part of the proof. We next turn to the second major case, $N<p \leq v$.

If $N \geq 1$ or $N=0$, we have $\left(m_{1}, \ldots, m_{v}\right) \neq 0$, and the lemma follows from the first part and Lemma 3.3. Otherwise, let $t^{\bar{m}} k_{p}=t_{0}^{m_{0}} k_{p}$. Set $\mathscr{L}_{\underline{0}}=\bigoplus_{m_{0} \in \mathbb{Z}} \mathbb{C} t_{0}^{m_{0}} d_{0} \oplus$ $\mathbb{C} k_{0}$ and $W=U\left(\mathscr{L}_{\underline{0}}\right) v$, where $v \in V_{\bar{s}}$ is a homogeneous element. Since $c_{0} \neq 0$, the sets $\left\{\operatorname{dim} W_{\left(n_{0}, 0\right)+\bar{s}} \mid n_{0} \in \mathbb{Z}\right\}$ are not uniformly bounded. But if neither $t_{0}^{m_{0}} k_{p}$ 
nor $t_{0}^{-m_{0}} k_{p}$ is locally nilpotent, then $t_{0} k_{p}$ and $t_{0}^{-1} k_{p}$ are not locally nilpotent. So by Lemmas 3.2 and 3.1, $\operatorname{dim} V_{\left(n_{0}, 0\right)+\bar{s}}=\operatorname{dim} V_{\bar{s}}$ for all $n_{0} \in \mathbb{Z}$, which is impossible since $\operatorname{dim} V_{\left(n_{0}, 0\right)+\bar{s}} \geq \operatorname{dim} W_{\left(n_{0}, 0\right)+\bar{s}}$. This proves Lemma 3.4

For $0 \leq p \leq N$, consider the direct sum

$$
\bigoplus_{m_{p} \in \mathbb{Z}} \mathbb{C} t_{p}^{m_{p}} d_{p} \oplus \mathbb{C} k_{p}
$$

which is a Virasoro Lie subalgebra of $\mathscr{L}$. Since $c_{p} \neq 0$, it follows from [Mathieu 1992] that there is a nonzero $v_{p} \in V_{\bar{r}}$ for some $\bar{r} \in \mathbb{Z}^{\nu+1}$ such that

$$
t_{p}^{m_{p}} d_{p} v_{p}=0 \text { for all } m_{p} \in \mathbb{Z}_{+}
$$

or

$$
t_{p}^{m_{p}} d_{p} v_{p}=0 \text { for all } m_{p} \in \mathbb{Z}_{-} .
$$

Lemma 3.5. If $v_{p} \in V_{\bar{r}}$ satisfies (3-6), the sets

$$
\left\{t_{p}^{m_{p}} k_{q} \mid m_{p} \in \mathbb{Z}_{+}, q=0,1,2, \ldots, v, q \neq p\right\}
$$

are all locally nilpotent on $V$. Likewise for (3-7), with $\mathbb{Z}_{+}$replaced by $\mathbb{Z}_{-}$.

Proof. We only prove the first statement. Suppose it is false; then by Lemma 3.3 $t_{p} k_{q}$ is not locally nilpotent on $V$ for some $q \in\{0,1, \ldots, v\}, q \neq p$. By Lemma $3.4, t_{p}^{-1} k_{q}$ is locally nilpotent. Therefore there exists $k \in \mathbb{Z}_{+}$such that

$$
\left(t_{p}^{-1} k_{q}\right)^{k-1} v_{p} \neq 0, \quad\left(t_{p}^{-1} k_{q}\right)^{k} v_{p}=0 .
$$

So

$$
\begin{aligned}
t_{p}^{2} d_{p}\left(t_{p}^{-1} k_{q}\right)^{k} v_{p} & =-k t_{p} k_{q}\left(t_{p}^{-1} k_{q}\right)^{k-1} v_{p}+\left(t_{p}^{-1} k_{q}\right)^{k} t_{p}^{2} d_{p} v_{p} \\
& =-k t_{p} k_{q}\left(t_{p}^{-1} k_{q}\right)^{k-1} v_{p}=0 .
\end{aligned}
$$

This implies that $t_{p} k_{q}$ is locally nilpotent, a contradiction.

Lemma 3.6. If $v_{p} \in V_{\bar{r}}$ satisfies (3-6), the sets

$$
\left\{t^{\bar{m}} k_{p} \mid \bar{m}=\left(m_{0}, \ldots, m_{v}\right) \in \mathbb{Z}^{v+1}, m_{p} \in \mathbb{Z}_{+}\right\}
$$

are all locally nilpotent on $V$. Likewise for (3-7), with $\mathbb{Z}_{+}$replaced by $\mathbb{Z}_{-}$.

Proof. Again we only prove the first statement. Without loss of generality, we assume that $p=0$. Let $\mathscr{K}^{\prime}$ be the subspace of $\mathscr{K}$ spanned by elements of $\mathscr{K}$ which are locally nilpotent on $V$. If $t^{\underline{m}} k_{0}$, for any $\underline{m} \in \mathbb{Z}^{\nu} \backslash\{0\}$, is not locally nilpotent on $V$, the lemma holds thanks to Lemmas 3.3 and 3.5. Suppose $\mathscr{K} \mathcal{K}^{\prime} \cap\left\{t \underline{m} k_{0} \mid \underline{m} \in \mathbb{Z}^{\nu}\right\} \neq\{0\}$. By Lemmas 3.2, 3.3 and 3.5, if $t^{\underline{m}} k_{0} \in \mathscr{K}^{\prime}$, then $t^{-\underline{m}} k_{0} \notin \mathscr{K}^{\prime}$, and $t_{0}^{m_{0}} t^{\underline{m}} k_{0} \in \mathscr{K}^{\prime}$ for all $m_{0}>0$.

Case 1: Suppose $t_{0}^{m_{0}} t^{-\underline{m}} k_{0} \in \mathscr{K}^{\prime}$ for any $t^{\underline{m}} k_{0} \in \mathscr{Y}^{\prime}$. Then the lemma is proved. 
Case 2: Suppose there exists $0 \neq t^{\underline{m}} k_{0} \in \mathscr{K}^{\prime}$ such that $t_{0} t^{-\underline{m}} k_{0} \notin \mathscr{K ^ { \prime }}$. Since $\underline{m}=$ $\left(m_{1}, \ldots, m_{v}\right) \neq 0$, we can assume that $m_{a} \neq 0$ for some $a \in\{1,2, \ldots, v\}$. Let $V_{\bar{r}_{0}}$ be such that

$$
\operatorname{dim} V_{\bar{r}_{0}}=\min \left\{\operatorname{dim} V_{\bar{s}} \mid V_{\bar{s}} \neq 0, \bar{s} \in \mathbb{Z}^{v+1}\right\} .
$$

Case 2.1: Assume $t_{0}^{i} t^{-\underline{m}} k_{0} \notin \mathscr{K}^{\prime}$ for any $i>0$. Let $l \in \mathbb{Z}_{+}$and consider

$$
\sum_{i=0}^{l} a_{i} t_{0}^{-i} t^{-\underline{m}} k_{0} t_{0}^{i} t^{-\underline{m}} k_{0} v=0,
$$

where $v \in V_{\bar{r}_{0}} \backslash\{0\}$. By Lemma 3.4, $\left\{t_{0}^{i} t^{\underline{m}} k_{0}, t_{0}^{-i} t^{\underline{m}} k_{0} \mid i \in \mathbb{Z}_{+}\right\} \subseteq \mathscr{K}^{\prime}$. So by Lemma 3.2 , we have

$$
t_{0}^{i} t^{\underline{m}} k_{0} V_{\bar{r}_{0}}=t_{0}^{-i} t^{\underline{m}} k_{0} V_{\bar{r}_{0}}=t_{0}^{i} t^{\underline{m}} d_{p} V_{\bar{r}_{0}}=t_{0}^{-i} t^{\underline{m}} d_{p} V_{\bar{r}_{0}}=0, i \in \mathbb{Z}_{+}, 0 \leq p \leq v .
$$

Let $j \in\{0,1, \ldots, l\}$. From (3-8) we have

$$
t_{0}^{-j} t^{\underline{m}} d_{a} t_{0}^{j} t^{\underline{m}} d_{a}\left(\sum_{i=0}^{l} a_{i} t_{0}^{-i} t^{-\underline{m}} k_{0} t_{0}^{i} t^{-\underline{m}} k_{0}\right) v=0 .
$$

Therefore

$$
\sum_{i=0}^{l} a_{i}\left(-m_{a}\right) t_{0}^{j-i} k_{0}\left(-m_{a}\right) t_{0}^{i-j} k_{0} v=a_{j} m_{a}^{2} c_{0}^{2} v=0 .
$$

So $a_{j}=0, j=0,1, \ldots, l$. This means $\left.\left\{t_{0}^{-i} t^{-\underline{m}} k_{0} t_{0}^{i} t^{-\underline{m}} k_{0}\right) v \mid 0 \leq i \leq l\right\}$ are linearly independent. Since $l$ can be any positive integer, it follows that $V_{\bar{r}_{0}-(0,2 \underline{m})}$ is infinite-dimensional, a contradiction.

Case 2.2: Assume there exists $l \in \mathbb{Z}_{+}$such that

$$
t_{0}^{l-1} t^{-\underline{m}} k_{0} \notin \mathscr{K ^ { \prime }}, \quad t_{0}^{l} t^{-\underline{m}} k_{0} \in \mathscr{K}^{\prime} .
$$

(I) Assume that $t_{0}^{l} t^{-i \underline{m}} k_{0} \in \mathscr{Y K}^{\prime}$ for any $i \in \mathbb{Z}_{+}$. Let $s>0$ and consider

$$
\sum_{i=1}^{s} a_{i} t_{0}^{-l} t^{i \underline{m}} k_{0} t^{-i \underline{m}} k_{0} v=0 .
$$

Similar to the proof above, we can deduce that $V_{\bar{r}_{0}-(l, 0)}$ is infinite-dimensional, in contradiction with the assumption that $V$ has finite-dimensional weight spaces.

(II) Assume there exists $s_{1} \in \mathbb{Z}_{+}$such that

$$
t_{0}^{l} t^{-\underline{m}} k_{0} \in \mathscr{K}^{\prime}, \quad t_{0}^{l} t^{-2 \underline{m}} k_{0} \in \mathscr{K}^{\prime}, \quad \ldots, \quad t_{0}^{l} t^{-s_{1}} \underline{\underline{m}} k_{0} \in \mathscr{K}^{\prime}, \quad t_{0}^{l} t^{-\left(s_{1}+1\right) \underline{m}} k_{0} \notin \mathscr{K}^{\prime} .
$$

Then there exist $s_{2}, s_{3}, \ldots, s_{k}, \ldots$ such that $s_{i} \geq s_{1}$ for $i=2,3, \ldots, k, \ldots$ and

$$
t_{0}^{i l} t^{\left(-s_{1}-s_{2}-\cdots-s_{i-1}-1\right) \underline{m}} k_{0} \in \mathscr{K}^{\prime}, t_{0}^{i l} t^{\left(-s_{1}-s_{2}-\cdots-s_{i-1}-2\right)} \underline{m} k_{0} \in \mathscr{K}^{\prime}, \ldots,
$$




$$
t_{0}^{i l} t^{\left(-s_{1}-s_{2}-\cdots-s_{i-1}-s_{i}\right)} \underline{m} k_{0} \in \mathscr{K}^{\prime}, t_{0}^{i l} t^{\left(-s_{1}-s_{2}-\cdots-s_{i-1}-s_{i}-1\right) \underline{m}} k_{0} \notin \mathscr{K}^{\prime} .
$$

Assume that

$$
\begin{aligned}
\left(\sum_{i=1}^{s_{1}} a_{i} t_{0}^{-l} t^{i \underline{m}} k_{0} t^{-i \underline{m}} k_{0}+\sum_{i=1}^{s_{2}} a_{s_{1}+i} t_{0}^{-2 l} t^{\left(s_{1}+i\right)} \underline{m} k_{0} t_{0}^{l} t^{-\left(s_{1}+i\right)} \underline{m}_{0}\right. \\
\quad+\sum_{i=1}^{s_{3}} a_{s_{1}+s_{2}+i} t_{0}^{-3 l} t^{\left(s_{1}+s_{2}+i\right) \underline{m}} k_{0} t_{0}^{2 l} t^{-\left(s_{1}+s_{2}+i\right)} \underline{m} k_{0}+\cdots \\
\left.\quad+\sum_{i=1}^{s_{k}} a_{s_{1}+\cdots+s_{k-1}+i} t_{0}^{-k l} t^{\left(s_{1}+\cdots+s_{k-1}+i\right)} \underline{m} k_{0} t_{0}^{(k-1) l} t^{-\left(s_{1}+\cdots+s_{k-1}+i\right) \underline{m}} k_{0}\right) v=0 .
\end{aligned}
$$

Let

$$
\begin{array}{ll}
t^{j \underline{m}} d_{a} t_{0}^{l} t^{-j \underline{m}} d_{a}, & 1 \leq j \leq s_{1}, \\
t_{0}^{-l} t^{\left(s_{1}+j\right) \underline{m}} d_{a} t_{0}^{2 l} t^{-\left(s_{1}+j\right)} \underline{m} d_{a}, & 1 \leq j \leq s_{2}, \\
\ldots, & \\
t_{0}^{-(k-1) l} t^{\left(s_{1}+s_{2}+\cdots+s_{k-1}+j\right)} \underline{m} d_{a} t_{0}^{k l} t^{-\left(s_{1}+s_{2}+\cdots+s_{k-1}+j\right)} \underline{m} d_{a}, & 1 \leq j \leq s_{k}
\end{array}
$$

act on the two sides of the above equation respectively. By Lemma 3.4, we deduce that $a_{i}=0$, for $i=1,2, \ldots, s_{1}$, and that

$$
a_{s_{1}+\cdots+s_{j-1}+i}=0 \quad \text { for } i=1,2, \ldots, s_{j}, 2 \leq j \leq k .
$$

Since $k$ can be any positive integer, it follows that $V_{\bar{r}_{0}-(l, \underline{0})}$ is infinite-dimensional, which contradicts our assumption. The lemma is proved.

Lemmas 3.1 through 3.6 immediately yield the following result.

Theorem 3.7. Let $V$ be an irreducible weight module of $\mathscr{L}$ such that $c_{0}, \ldots, c_{N}$ are $\mathbb{Z}$-linearly independent and $N \geq 1$. Then $V$ has weight spaces that are infinitedimensional.

Let

$$
\begin{aligned}
& \mathscr{L}_{+}=\sum_{p=0}^{v} t_{0} \mathbb{C}\left[t_{0}, t_{1}^{ \pm 1}, \ldots, t_{v}^{ \pm 1}\right] k_{p} \oplus \sum_{p=0}^{v} t_{0} \mathbb{C}\left[t_{0}, t_{1}^{ \pm 1}, \ldots, t_{v}^{ \pm 1}\right] d_{p}, \\
& \mathscr{L}_{-}=\sum_{p=0}^{v} t_{0}^{-1} \mathbb{C}\left[t_{0}^{-1}, t_{1}^{ \pm 1}, \ldots, t_{v}^{ \pm 1}\right] k_{p} \oplus \sum_{p=0}^{v} t_{0}^{-1} \mathbb{C}\left[t_{0}^{-1}, t_{1}^{ \pm 1}, \ldots, t_{v}^{ \pm 1}\right] d_{p}, \\
& \mathscr{L}_{0}=\sum_{p=0}^{v} \mathbb{C}\left[t_{1}^{ \pm 1}, \ldots, t_{v}^{ \pm 1}\right] k_{p} \oplus \sum_{p=0}^{v} \mathbb{C}\left[t_{1}^{ \pm 1}, \ldots, t_{v}^{ \pm 1}\right] d_{p} .
\end{aligned}
$$

Then

$$
\mathscr{L}=\mathscr{L}_{+} \oplus \mathscr{L}_{0} \oplus \mathscr{L}_{-} .
$$


Definition 3.8. Let $W$ be a weight module of $\mathscr{L}$. If there is a nonzero vector $v_{0} \in W$ such that

$$
\mathscr{L}_{+} v_{0}=0, W=U(\mathscr{L}) v_{0},
$$

then $W$ is called a highest weight module of $\mathscr{L}$. If there is a nonzero vector $v_{0} \in W$ such that

$$
\mathscr{L}_{-} v_{0}=0, W=U(\mathscr{L}) v_{0},
$$

then $W$ is called a lowest weight module of $\mathscr{L}$.

From Lemmas 3.2 and 3.6, we obtain:

Theorem 3.9. Let $V$ be an irreducible weight module of $\mathscr{L}$ with finite-dimensional weight spaces and with central charges $c_{0} \neq 0, c_{1}=c_{2}=\cdots=c_{v}=0$. Then $V$ is a highest or lowest weight module of $\mathscr{L}$.

In the remainder of this section we assume that $V$ is an irreducible weight module of $\mathscr{L}$ with finite-dimensional weight spaces and with central charges $c_{0} \neq 0$, $c_{1}=\cdots=c_{\nu}=0$.

Set

$$
T= \begin{cases}\left\{v \in V \mid \mathscr{L}_{+} v=0\right\} & \text { if } V \text { is a highest weight module of } \mathscr{L}, \\ \left\{v \in V \mid \mathscr{L}_{-} v=0\right\} & \text { if } V \text { is a lowest weight module of } \mathscr{L} .\end{cases}
$$

Then $T$ is a $\mathscr{L}_{0}$-module and

$$
V=U\left(\mathscr{L}_{-}\right) T \quad \text { or } \quad V=U\left(\mathscr{L}_{+}\right) T .
$$

Since $V$ is an irreducible $\mathscr{L}$-module, $T$ is an irreducible $\mathscr{L}_{0}$-module. $T$ has the decomposition

$$
T=\bigoplus_{\underline{m} \in \mathbb{Z}^{v}} T_{\underline{m}},
$$

where $\underline{m}=\left(m_{1}, m_{2}, \ldots, m_{v}\right), T_{\underline{m}}=\left\{v \in T \mid d_{i} v=\left(m_{i}+\mu\left(d_{i}\right)\right) v, 1 \leq i \leq v\right\}$ and $\mu$ is a fixed weight of $T$. As in the proof in [Jiang and Meng 2003; Eswara Rao and Jiang 2005], we can deduce:

Theorem 3.10. (1) For all $\underline{m}, \underline{n} \in \mathbb{Z}^{v}, p=1,2, \ldots, v$, we have

$$
\begin{aligned}
\operatorname{dim} T_{\underline{m}} & =\operatorname{dim} T_{\underline{n}}, t^{\underline{m}} k_{p} \cdot T=0, \\
t^{\underline{m}} k_{0}\left(v_{1}(\underline{n}), \ldots, v_{m}(\underline{n})\right) & =c_{0}\left(v_{1}(\underline{m}+\underline{n}), v_{2}(\underline{m}+\underline{n}), \ldots, v_{n}(\underline{m}+\underline{n})\right), \\
t^{\underline{m}} d_{0}\left(v_{1}(\underline{n}), v_{2}(\underline{n}), \ldots, v_{n}(\underline{n})\right) & =\mu\left(d_{0}\right)\left(v_{1}(\underline{m}+\underline{n}), v_{2}(\underline{m}+\underline{n}), \ldots, v_{n}(\underline{m}+\underline{n})\right),
\end{aligned}
$$

where $\left\{v_{1}(\underline{0}), \ldots, v_{m}(\underline{0})\right\}$ is a basis of $T_{\underline{0}}$ and $v_{i}(\underline{m})=\frac{1}{c_{0}} t \underline{m} k_{0} v_{i}(\underline{0})$, for $i=1,2$, $\ldots, m$. 
(2) As an $\left(\mathscr{A}_{v} \oplus \mathscr{D}_{v}\right)$-module, $T$ is isomorphic to

$$
F^{\alpha}(\psi, b)=V(\psi, b) \otimes \mathbb{C}\left[t_{1}^{ \pm 1}, \ldots, t_{v}^{ \pm 1}\right]
$$

for some $\alpha=\left(\alpha_{1}, \ldots, \alpha_{v}\right), \psi$, and $b$, where $\mathscr{A}_{v}=\mathbb{C}\left[t_{1}^{ \pm 1}, \ldots, t_{v}^{ \pm 1}\right], \mathscr{D}_{v}$ is the derivation algebra of $\mathscr{A}_{v}$, and $V(\psi, b)$ is an m-dimensional, irreducible $g l_{v}(\mathbb{C})$-module satisfying $\psi(I)=b \operatorname{id}_{V(\psi, b)}$ and

for $w \in V(\psi, b)$.

$$
t^{\underline{r}} d_{p}\left(w \otimes t^{\underline{m}}\right)=\left(m_{p}+\alpha_{p}\right) w \otimes t^{\underline{r} \underline{\underline{m}}}+\sum_{i=1}^{v} r_{i} \psi\left(E_{i p}\right) w \otimes t^{\underline{r}+\underline{m}}
$$

Let

$$
M=\operatorname{Ind}_{\mathscr{L}_{+}+\mathscr{L}_{0}}^{\mathscr{L}} T \quad \text { or } \quad M=\operatorname{Ind}_{\mathscr{L}_{-}+\mathscr{L}_{0}}^{\mathscr{L}} T .
$$

Theorem 3.11. Among the submodules of $M$ intersecting $T$ trivially, there is a maximal one, which we denote by $M^{\mathrm{rad}}$. Moreover $V \cong M / M^{\mathrm{rad}}$.

\section{The structure of $V$ with $c_{0}=\cdots=c_{v}=0$}

Assume that $V$ is an irreducible weight module of $\mathscr{L}$ with finite-dimensional weight spaces and $c_{0}=\cdots=c_{\nu}=0$.

Lemma 4.1. For any $t^{\bar{r}} k_{p} \in \mathscr{K}, t^{\bar{r}} k_{p}$ or $t^{-\bar{r}} k_{p}$ is locally nilpotent on $V$.

Lemma 4.2. If $V$ is uniformly bounded, $t^{\bar{r}} k_{p}$ is locally nilpotent on $V$ for any $t^{\bar{r}} k_{p} \in \mathcal{K}$.

Proof. For $t^{\bar{r}} k_{p} \in \mathscr{K}$, by Lemma 4.1, $t^{\bar{r}} k_{p}$ or $t^{-\bar{r}} k_{p}$ is nilpotent on $V_{\bar{m}}$ for all $\bar{m} \in \mathbb{Z}^{v+1}$. Since $V$ is uniformly bounded, i.e., $\max \left\{\operatorname{dim} V_{\bar{m}} \mid \bar{m} \in \mathbb{Z}^{\nu+1}\right\}<\infty$, there exists $N \in \mathbb{Z}_{+}$such that

$$
\left(t^{\bar{r}} k_{p} t^{-\bar{r}} k_{p}\right)^{N} V=0,\left(t^{\bar{r}} k_{p} t^{-\bar{r}} k_{p}\right)^{N-1} V \neq 0
$$

If the lemma is false, we can assume that $t^{-\bar{r}} k_{p}$ is not locally nilpotent on $V$. Therefore for any $0 \neq v \in V$, we have $t^{-\bar{r}} k_{p} v \neq 0$. So

$$
\left(t^{\bar{r}} k_{p}\right)^{N} V=0 .
$$

Let $t^{-2 \bar{r}} d_{q} \in \mathscr{K}$ be such that $p \neq q$ and $r_{q} \neq 0$. By the fact that $\left[t^{-2 \bar{r}} d_{q}, t^{\bar{r}} k_{p}\right]=$ $r_{q} t^{-\bar{r}} k_{p}$, we deduce that $t^{-\bar{r}} k_{p}\left(t^{\bar{r}} k_{p}\right)^{N-1} V=0$, a contradiction.

Lemma 4.3. If there exists $0 \neq v \in V$ such that $t^{\bar{m}} k_{p} v=0$ for all $\bar{m} \in \mathbb{Z}^{v+1}$ and $0 \leq p \leq v$. Then $\mathscr{K}(V)=0$.

Proof. This follows from (2-2), since $\mathscr{K}$ is commutative and $V$ is an irreducible $\mathscr{L}$-module.

Theorem 4.4. If $V$ is uniformly bounded, $t^{\bar{r}} k_{p} V$ vanishes for any $t^{\bar{r}} k_{p} \in \mathscr{K}$. 
Proof. Let $0 \neq t_{i} k_{p} \in \mathscr{K}$. If $t_{i} k_{p} V=0$, it is easy to prove that $\mathscr{K}(V)=0$. If $t_{i} k_{p} V \neq 0$. Since $V$ is uniformly bounded, by Lemma 4.2, there exists $l \in \mathbb{Z}_{+}$such that

$$
\left(t_{i} k_{p} t_{i}^{-1} k_{p}\right)^{l} V=0, \quad\left(t_{1} k_{p} t_{1}^{-1} k_{p}\right)^{l-1} V \neq 0 .
$$

If there exists $s \in \mathbb{Z}_{+}$such that $\left(t_{i}^{-1} k_{p}\right)^{s} V=0,\left(t_{i}^{-1} k_{p}\right)^{s-1} V \neq 0$. By the fact that $\left[t^{\bar{m}} d_{i}, t_{i}^{-1} k_{p}\right]=-t_{i}^{-1} t^{\bar{m}} k_{p}$ and $\left[t^{\bar{m}} d_{p}, t_{i}^{-1} k_{p}\right]=t_{i}^{-1} t^{\bar{m}} k_{i}$, we have

$$
t^{\bar{r}} k_{p}\left(t_{i}^{-1} k_{p}\right)^{s-1} V=t^{\bar{r}} k_{i}\left(t^{-\bar{r}} k_{p}\right)^{s-1} V=0 \text { for all } \bar{r} \in \mathbb{Z}^{v+1} \text {. }
$$

If $\left(t_{i}^{-1} k_{p}\right)^{s} V \neq 0$ for all $s \in \mathbb{Z}_{+}$. Then by (4-1) there is $r \geq 0$ such that $\left(t_{i} k_{p}\right)^{l-i}\left(t_{i}^{-1} k_{p}\right)^{l+i} V=0$ for all $0 \leq i \leq r$, and $\left(t_{i} k_{p}\right)^{l-r-1}\left(t_{i}^{-1} k_{p}\right)^{l+r+1} V \neq 0$. So for any $\bar{m} \in \mathbb{Z}^{\nu+1}$, we have

$$
t^{-\bar{m}} d_{i}\left(t_{i} k_{p}\right)^{l-r}\left(t_{i}^{-1} k_{p}\right)^{l+r+1} V=0, \quad t^{-\bar{m}} d_{p}\left(t_{i} k_{p}\right)^{l-r}\left(t_{i}^{-1} k_{p}\right)^{l+r+1} V=0 .
$$

Therefore

$$
\begin{aligned}
t^{\bar{r}} k_{p}\left(t_{i} k_{p}\right)^{l-r-1}\left(t_{i}^{-1} k_{p}\right)^{l+r+1} V & =0, \\
t^{\bar{r}} k_{i}\left(t_{i} k_{p}\right)^{l-r-1}\left(t_{i}^{-1} k_{p}\right)^{l+r+1} V & =0,
\end{aligned}
$$

for all $\bar{r} \in \mathbb{Z}^{v+1}$.

Case 1: $v \in 2 \mathbb{Z}_{+}+1$. By the preceding discussion, there exist nonnegative integers $l_{i}$ and $r_{i}$, for $i=0,2,4, \ldots, v-1$, such that

$$
\left(t_{v} k_{v-1}\right)^{l_{v-1}}\left(t_{v}^{-1} k_{\nu-1}\right)^{r_{v-1}}\left(t_{v-2} k_{\nu-3}\right)^{l_{v-3}}\left(t_{\nu-2}^{-1} k_{v-3}\right)^{r_{v-3}} \cdots\left(t_{1} k_{0}\right)^{l_{0}}\left(t_{1}^{-1} k_{0}\right)^{r_{0}} V \neq 0
$$

and

$$
t^{\bar{m}} k_{p}\left(t_{\nu} k_{\nu-1}\right)^{l_{\nu-1}}\left(t_{v}^{-1} k_{v-1}\right)^{r_{\nu-1}}\left(t_{\nu-2} k_{\nu-3}\right)^{l_{\nu-3}}\left(t_{\nu-2}^{-1} k_{\nu-3}\right)^{r_{\nu-3}} \cdots\left(t_{1} k_{0}\right)^{l_{0}}\left(t_{1}^{-1} k_{0}\right)^{r_{0}} V
$$

vanishes for all $0 \leq p \leq v$ and $\bar{m} \in \mathbb{Z}^{v+1}$. By Lemma 4.3, the conclusion of the theorem holds.

$\underline{\text { Case 2: }} v \in 2 \mathbb{Z}$. Then there exist nonnegative integers $l_{i}$ and $r_{i}$, for $i=0,2,4, \ldots$, $v-2$, such that

$$
W=\left(t_{\nu-1} k_{\nu-2}\right)^{l_{\nu-2}}\left(t_{\nu-1}^{-1} k_{\nu-2}\right)^{r_{\nu-2}}\left(t_{\nu-3} k_{\nu-4}\right)^{l_{\nu-4}}\left(t_{\nu-3}^{-1} k_{\nu-4}\right)^{r_{\nu-4}} \cdots\left(t_{1} k_{0}\right)^{l_{0}}\left(t_{1}^{-1} k_{0}\right)^{r_{0}} V
$$

is nonzero and

$$
t^{\bar{m}} k_{p} W=0
$$

for all $0 \leq p \leq v-1$ and $\bar{m} \in \mathbb{Z}^{v+1}$. By (2-1), we know that

$$
t^{\bar{m}} k_{v} W=0 \text {, }
$$


for $\bar{m} \in \mathbb{Z}^{v+1}$ such that $m_{v} \neq 0$. If there exists $t^{\bar{r}_{0}} k_{v}$ satisfying $t^{\bar{r}_{0}} k_{v} W \neq 0$, let

$$
\begin{aligned}
\mathscr{L}_{v} & =\operatorname{span}\left\{t^{\underline{m}} d_{i}, t^{\bar{m}} d_{\nu}, t^{\underline{m}} k_{\nu} \mid t^{\underline{m}}=t_{0}^{m_{0}} t_{1}^{m_{1}} \cdots t_{\nu-1}^{m_{\nu-1}}, 0 \leq i \leq v-1,\right. \\
\underline{m} & \left.=\left(m_{0}, \ldots, m_{\nu-1}\right) \in \mathbb{Z}^{v}, \bar{m} \in \mathbb{Z}^{v+1}\right\}, \\
W^{\prime} & =U\left(\mathscr{L}_{\nu}\right) W .
\end{aligned}
$$

Then $W^{\prime} \neq 0$ and

$$
t^{\bar{m}} k_{p} W^{\prime}=0, \quad t^{\bar{n}} k_{v} W^{\prime}=0,
$$

for all $0 \leq p \leq v-1, \bar{m} \in \mathbb{Z}^{v+1}$, and $\bar{n} \in \mathbb{Z}^{v+1}$ such that $n_{v} \neq 0$. If there exists $0 \neq t^{\underline{m}} k_{v}$ such that $t^{\underline{m}} k_{v} W^{\prime} \neq 0$, we have

$$
\left(t^{-\underline{m}} k_{\nu}\right)^{l}\left(t^{\underline{m}} k_{\nu}\right)^{l} W^{\prime}=0 \quad \text { and } \quad\left(t^{-\underline{m}} k_{\nu}\right)^{l-1}\left(t^{\underline{m}} k_{v}\right)^{l-1} W^{\prime} \neq 0
$$

for some $l \in \mathbb{Z}_{+}$. As in the preceding proof, we can deduce that there exists a nonzero $v \in W^{\prime}$ such that

$$
t^{\underline{n}} k_{v} v=0
$$

for all $\underline{n} \in \mathbb{Z}^{v}$. Therefore

$$
t^{\bar{m}} k_{p} v=0
$$

for all $\bar{m} \in \mathbb{Z}^{v+1}$ and $0 \leq p \leq v$. We have proved that $\mathscr{K}(V)=0$.

\section{References}

[Belavin et al. 1984] A. A. Belavin, A. M. Polyakov, and A. B. Zamolodchikov, "Infinite conformal symmetry in two-dimensional quantum field theory", Nuclear Phys. B 241:2 (1984), 333-380. MR 86m:81097 Zbl 0661.17013

[Berman and Billig 1999] S. Berman and Y. Billig, "Irreducible representations for toroidal Lie algebras", J. Algebra 221:1 (1999), 188-231. MR 2000k:17004 Zbl 0942.17016

[Billig 2003] Y. Billig, "Abelian extensions of the group of diffeomorphisms of a torus", Lett. Math. Phys. 64:2 (2003), 155-169. MR 2004h:22012 Zbl 1079.58004

[Chari and Pressley 1988] V. Chari and A. Pressley, "Unitary representations of the Virasoro algebra and a conjecture of Kac", Compositio Math. 67:3 (1988), 315-342. MR 89h:17025 Zbl 0661.17022

[Di Francesco et al. 1997] P. Di Francesco, P. Mathieu, and D. Sénéchal, Conformal field theory, Springer, New York, 1997. MR 97g:81062 Zbl 0869.53052

[Dotsenko and Fateev 1984] V. S. Dotsenko and V. A. Fateev, "Conformal algebra and multipoint correlation functions in 2D statistical models", Nuclear Phys. B 240:3 (1984), 312-348. MR 85i: 82061

[Eswara Rao 2004] S. Eswara Rao, "Partial classification of modules for Lie algebra of diffeomorphisms of $d$-dimensional torus", J. Math. Phys. 45:8 (2004), 3322-3333. MR 2005d:17028 Zbl 1071.17020

[Eswara Rao and Jiang 2005] S. Eswara Rao and C. Jiang, "Classification of irreducible integrable representations for the full toroidal Lie algebras", J. Pure Appl. Algebra 200:1-2 (2005), 71-85. MR 2006b:17038 Zbl 1070.17009 
[Eswara Rao and Moody 1994] S. Eswara Rao and R. V. Moody, "Vertex representations for $n$ toroidal Lie algebras and a generalization of the Virasoro algebra", Comm. Math. Phys. 159:2 (1994), 239-264. MR 94m:17028 Zbl 0808.17018

[Jiang and Meng 2003] C. Jiang and D. Meng, "Integrable representations for generalized Virasorotoroidal Lie algebras”, J. Algebra 270:1 (2003), 307-334. MR 2005b:17053 Zbl 1037.17029

[Kac 1982] V. G. Kac, "Some problems on infinite-dimensional Lie algebras and their representations", pp. 117-126 in Lie algebras and related topics (New Brunswick, NJ, 1981), edited by D. J. Winter, Lecture Notes in Math. 933, Springer, Berlin, 1982. MR 84e:17010 Zbl 0493.17011

[Kaplansky and Santharoubane 1985] I. Kaplansky and L. J. Santharoubane, "Harish-Chandra modules over the Virasoro algebra", pp. 217-231 in Infinite dimensional groups with applications (Berkeley, 1984), edited by V. Kac, Math. Sci. Res. Inst. Publ. 4, Springer, New York, 1985. MR 87d:17013 Zbl 0589.17013

[Larsson 1992] T. A. Larsson, "Conformal fields: a class of representations of $\operatorname{Vect}(N)$ ", Internat. J. Modern Phys. A 7:26 (1992), 6493-6508. MR 93h:17053 Zbl 0972.17502

[Mathieu 1992] O. Mathieu, "Classification of Harish-Chandra modules over the Virasoro Lie algebra”, Invent. Math. 107:2 (1992), 225-234. MR 93d:17034 Zbl 0779.17025

[Ramos et al. 1990] E. Ramos, C.-H. Sah, and R. E. Shrock, "Algebras of diffeomorphisms of the N-torus”, J. Math. Phys. 31:8 (1990), 1805-1816. MR 91j:17043 Zbl 0733.17014

Received December 3, 2005.

\author{
CUIPO JIANG \\ DEPARTMENT OF MATHEMATICS \\ SHANGHAI JIAOTONG UNIVERSITY \\ SHANGHAI 200030 \\ CHINA \\ cpjiang@sjtu.edu.cn \\ QIFEN JIANG \\ DEPARTMENT OF MATHEMATICS \\ SHANGHAi JiAOTONG UNIVERSITY \\ SHANGHAI 200030 \\ CHINA \\ qfjiang@sjtu.edu.cn
}

